\title{
Application of Extended Permeability to a Thick Palladium Membrane
}

\author{
Shigeki Hara ${ }^{a,{ }^{*}}$, Misaki Ishitsuka ${ }^{\mathrm{b}}$, Hiroyuki Suda ${ }^{\mathrm{c}}$, Masakazu Mukaida $^{\mathrm{d}}$ and \\ Kenji Haraya ${ }^{\mathrm{e}}$
}

Research Institute for Innovation in Sustainable Chemistry, National Institute of Advanced Industrial Science and Technology (AIST), Central 5, Tsukuba 305-8565, Japan

as.hara@aist.go.jp, ${ }^{b}$ ishitsuka-m@aist.go.jp, ${ }^{c h}$.suda@aist.go.jp, ${ }^{d}$ mskz.mukaida@aist.go.jp, ek.haraya@aist.go.jp

Keywords: hydrogen, membrane, palladium, permeability, square-root law

\begin{abstract}
Some dense metal membranes are permeable only to hydrogen, useful to produce and purify hydrogen. Conventionally, hydrogen permeation flux through metal membranes is described as the square-root law. The permeability defined in the law is commonly used as a measure of membrane material. However, deviation from the law has been widely reported. We have extended the definition of permeability for precise description. This study applied it to a thick palladium membrane down to $0.01 \mathrm{MPa}$ in absolute pressure. Experimental results showed that hydrogen permeation flux through the palladium membrane $200 \mu \mathrm{m}$ thick did not obey the square-root law completely. From the permeation behavior, pressure-dependent permeability was evaluated. The resultant permeability was found to decrease and become a constant value, or intrinsic permeability, as pressure approached vacuum.
\end{abstract}

\section{Introduction}

Palladium membranes are permeable only to hydrogen. Therefore, they are expected to produce and purify hydrogen widely not only in industry but also for fuel cells. However, palladium is a platinum group metal, precious and limited in world production. To reduce palladium necessary for membranes, thin membranes and alternative membrane metals have to be developed.

Characterization of permeation behavior is the base in these developments. Conventionally, hydrogen permeation flux through metal membranes, $J\left[\mathrm{~mol} \mathrm{H}_{2} / \mathrm{m}^{2} \mathrm{~s}\right]$, is described as the following square-root law:

$$
J=\frac{\phi}{l}\left(\sqrt{p^{\mathrm{f}}}-\sqrt{p^{\mathrm{p}}}\right),
$$

where $l[\mathrm{~m}], p^{\mathrm{f}}[\mathrm{Pa}]$, and $p^{\mathrm{p}}[\mathrm{Pa}]$ are membrane thickness, feed-side, and permeation-side hydrogen pressures, respectively. The permeability, $\phi\left[\mathrm{mol} \mathrm{H}_{2} / \mathrm{m} \mathrm{s} \mathrm{Pa}^{0.5}\right]$, is commonly used as a measure of membrane material. However, deviation from the law has been widely reported [1-5].

To describe permeation behavior not obeying this law, a power law is often employed, where $n$-th power is used instead of the square root. Nevertheless, the power law has no theoretical base to analyze the permeation behavior relating other chemical properties including solubility and diffusivity.

To overcome the problem, we have extended the definition of permeability for precise description and analysis [6]. The definition has been proved mainly through permeation experiment for a 50- $\mu \mathrm{m}$-thick palladium membrane at atmospheric pressure or higher. This study applies it to a thick palladium membrane down to $0.01 \mathrm{MPa}$ to confirm its validity under wider conditions. 


\section{Theory}

The extended definition introduces differential and integral in the conventional square-root law, resulting in pressure-dependent permeability. Basic assumptions are a uniform flat membrane, diffusion controlling, and steady state.

The permeability is defined as a local permeability for small pressure difference in the following form:

$$
\phi(p)_{p=p^{\mathrm{p}}}=\lim _{p^{\mathrm{f}} \rightarrow p^{\mathrm{p}}} \frac{l J}{\sqrt{p^{\mathrm{f}}}-\sqrt{p^{\mathrm{p}}}} .
$$

The resultant permeability depends on pressure. This form is mathematically equivalent to the description under a constant permeation-side pressure:

$$
\phi(p)_{p=p^{\mathrm{f}}}=\frac{\mathrm{d}(l J)}{\mathrm{d} \sqrt{p^{\mathrm{f}}}} .
$$

These definitions are explained in detail in a previous report[6].

Conventional square-root law, power law and extended law in this study are compared in Table 1. Conventional solution coefficient, $K\left[\mathrm{~mol} \mathrm{H} / \mathrm{m}^{3} \mathrm{~Pa}^{0.5}\right]$, is defined from relation between hydrogen concentration in the metal, $C\left[\mathrm{~mol} \mathrm{H} / \mathrm{m}^{3}\right]$, and equilibrium hydrogen pressure. This coefficient is proportional to the equilibrium constant of reaction $\mathrm{H}_{2} \leftrightarrow 2 \mathrm{H}$ (in metal) taking Henry's law into account. The coefficient is also extended so as to apply to any behavior, e.g., even under high hydrogen concentration in the membrane metal where Henry's law is invalid.

On the other hand, the power law can often describe permeation behavior more precisely than the square-root law. Permeability for the power law, $\phi$ $\left[\mathrm{mol} \mathrm{H}_{2} / \mathrm{m} \mathrm{s} \mathrm{Pa}^{n}\right.$, and the power $n$ both are fitting parameters. However, thus determined permeability is usually difficult to analyze connecting diffusion and solution behavior due to lack of theoretical base. The permeability could not be compared directly with other permeability with different $n$. The extended law can deal with the power law also, that is, the power low can be converted to the extended law using the following formula[6]:

$$
\phi(p)=2 n \phi^{\prime} p^{n-0.5} \text {. }
$$

\begin{tabular}{|c|c|c|c|}
\hline & $\begin{array}{l}\text { Square-root law } \\
\text { (conventional) }\end{array}$ & $\begin{array}{l}\text { Power law } \\
\text { (often used) }\end{array}$ & $\begin{array}{l}\text { Extended law } \\
\text { (this study) }\end{array}$ \\
\hline Definition of permeability & $\phi=\frac{l J}{\sqrt{p^{\mathrm{f}}}-\sqrt{p^{\mathrm{p}}}}$ & $\phi^{\prime}=\frac{l J}{\left(p^{\mathrm{f}}\right)^{n}-\left(p^{\mathrm{p}}\right)^{n}}$ & $\phi(p)_{p=p^{\mathrm{f}}}=\frac{\mathrm{d}(l J)}{\mathrm{d} \sqrt{p^{\mathrm{f}}}}$ \\
\hline Flux estimation & $J=\frac{\phi}{l}\left(\sqrt{p^{\mathrm{f}}}-\sqrt{p^{\mathrm{p}}}\right.$ & $J=\frac{\phi^{\prime}}{l}\left\{\left(p^{\mathrm{f}}\right)^{n}-\left(p^{\mathrm{p}}\right)^{n}\right\}$ & $J=\frac{1}{l} \int_{\sqrt{p^{\mathrm{p}}}}^{\sqrt{p^{\mathrm{f}}}} \phi(p) \mathrm{d} \sqrt{p}$ \\
\hline $\begin{array}{l}\text { Relation with solution and } \\
\text { diffusion coefficients }\end{array}$ & $\phi=\frac{D K}{2}$ & & $\phi(p)=\frac{D(p) K(p)}{2}$ \\
\hline \multirow{2}{*}{$\begin{array}{l}\text { Definition } \\
\text { coefficient }\end{array}$} & $K=\frac{C}{\sqrt{p}}$ & & $K(p)=\frac{\mathrm{d} C}{\mathrm{~d} \sqrt{p}}$ \\
\hline & Henry's law & no theoretical base & $\begin{array}{c}\text { applicable to any } \\
\text { behavior }\end{array}$ \\
\hline
\end{tabular}

Table 1 Comparison among different permeability definitions 


\section{Experimental}

Experimental set-up is schematically drawn in Fig. 1. A sheet of pure palladium foil purchased from Ishifuku Metal Industry Co., Ltd. was used as a hydrogen permeable membrane. It was mounted in a hydrogen permeation cell. Pure hydrogen was introduced on both sides of the membrane: one was feed side and the other was permeation side. Feed-side pressure was changed from $0.01-1.0$

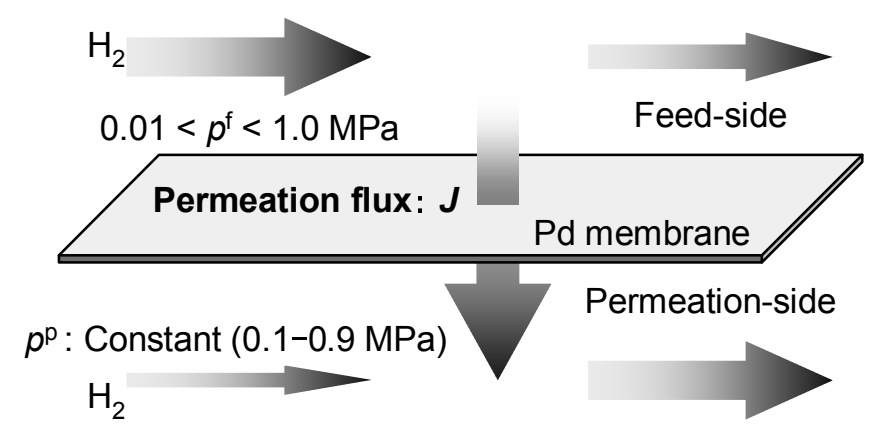

Fig. 1 Permeation test set-up $\mathrm{MPa}$ in absolute pressure while permeation-side pressure was fixed during each series of permeation tests. Several series were carried out for different permeation-side pressures. A rotary vacuum pump was used to reduce the feed-side pressure below atmospheric pressure. Hydrogen flow rate from the permeation side was measured by a mass flow meter, calibrated in prior using a soap flow meter. Permeation flux was estimated as the difference in flow rate between in the inlet and outlet of the permeation side. Operating conditions are summarized in Table 2.

Table 2 Operating conditions for permeation test

\begin{tabular}{ll}
\hline Membrane & Uniform pure palladium foil \\
Membrane thickness & $200 \mu \mathrm{m}$ \\
Effective permeation area & $2.98 \mathrm{~cm}^{2}$ \\
Feed-side gas & $99.99999 \%$ hydrogen \\
Feed-side pressure & $0.01-1.0 \mathrm{MPa}$ \\
Permeation-side gas & $99.99999 \%$ hydrogen \\
Permeation-side pressure & $0.1,0.5$, and $0.9 \mathrm{MPa}$ \\
Permeation temperature & $533-773 \mathrm{~K}$ \\
\hline
\end{tabular}

\section{Results and Discussion}

Deviation from Square-Root Law. Figure 2 depicts hydrogen permeation behavior around atmospheric permeation-side pressure in the conventional manner, i.e., as a function of the difference of the square roots of pressures on both sides of the membrane. Hydrogen permeation flux is almost proportional to the square-root pressure difference as widely known. However, deviation from the proportionality is found. The deviation is the greater at the lower temperatures.

Deviation from the square-root law is often seen for composite membranes, attributable to surface reaction controlling [7]. However, the deviation in this study is not caused by the surface reaction because the membrane is thick enough $(200 \mu \mathrm{m})$. Hydrogen permeation rate is much lower than those of composite membranes, so that hydrogen atom diffusion in the membrane must control the permeation rate.

Evaluation of Extended Permeability. For analysis, permeation flux is drawn as a function of the square root of feed-side pressure, $\sqrt{p^{\mathrm{f}}}$, for each constant permeation-side pressure. Figure 3 is an example.

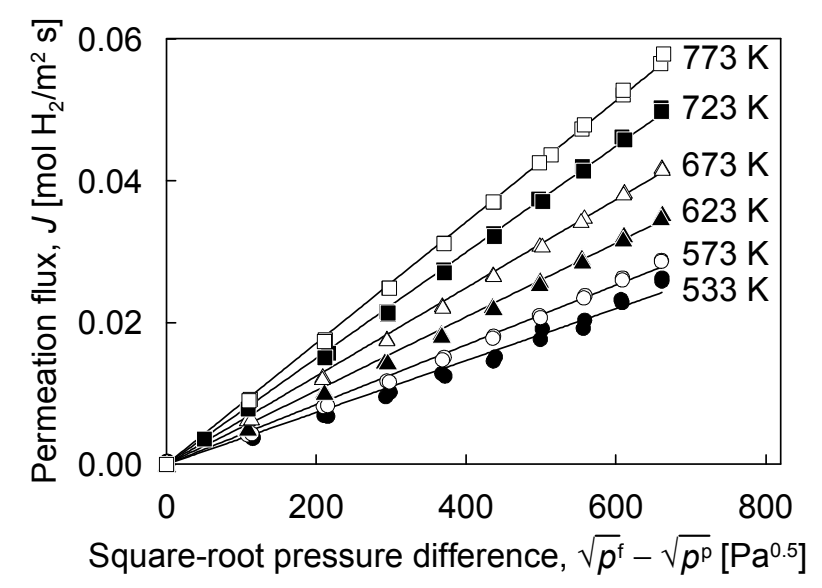

Fig. 2 Hydrogen permeation flux vs. the square root difference between feed- and permeationside pressures. 
According to Eq. 2, permeability can be determined as the slope where feed- and permeation-side pressures are the same, that is, no permeation. Three permeability values are derived from Fig. 3, indicated in Fig. 4 with open circles. Similarly, the other keys in the figure were determined.

Furthermore, a continuous function of permeability can be derived by Eq. 3 as described below. Each series in Fig. 3 can be fitted well using a polynomial, i.e., in the following form:

$$
J\left(\sqrt{p^{\mathrm{f}}}\right)=a\left(\sqrt{p^{\mathrm{f}}}\right)^{3}+b\left(\sqrt{p^{\mathrm{f}}}\right)^{2}+c \sqrt{p^{\mathrm{f}}}+d,
$$

where $a, b, c$, and $d$ are fitting parameters. Substituting this fitted function into Eq. 3, permeability is derived as a function of $\sqrt{p}$ :

$$
\phi(\sqrt{p})=l\left\{3 a(\sqrt{p})^{2}+2 b \sqrt{p}+c\right\} .
$$

Because these shapes in Fig. 3 are the same each other, the same $a$ is used for all three series of different $p^{\mathrm{p}}$ during this fitting procedure, as well as $b$ and $c$.

Equation 6 and also Fig. 4 do not distinguish $p^{\mathrm{f}}$ and $p^{\mathrm{p}}$ any more, just using $p$. This is because to distinguish both has no sense in $\phi$ of Eq. 2, equivalent to Eq. 3, where $p^{\mathrm{f}}=p^{\mathrm{p}}$. The reference [6] explains more in detail.

Functions determined in this manner are drawn with curves in Fig. 4. Keys and curves are well consistent each other, experimentally assuring that these two procedures are equivalent. The permeability decreases with decreasing pressure. The pressure dependency is the smaller at the lower pressures and the higher temperatures. These characteristics are the same as those for the $50 \mu \mathrm{m}$ membrane reported previously [6].

It should be noted that permeability becomes a constant value as pressure approaches vacuum, called as intrinsic permeability, hereafter. The behavior is reasonable because Henry's law is valid at low pressures due to low hydrogen concentrations. Diffusion coefficient may be regarded as a constant at low concentrations, as well. Therefore, square-root law is valid, that is, permeability may be written as a constant independent of pressure.

Arrhenius plot of intrinsic permeability is illustrated in Fig. 5. The experimental data are well align on a straight line, giving pre-exponential

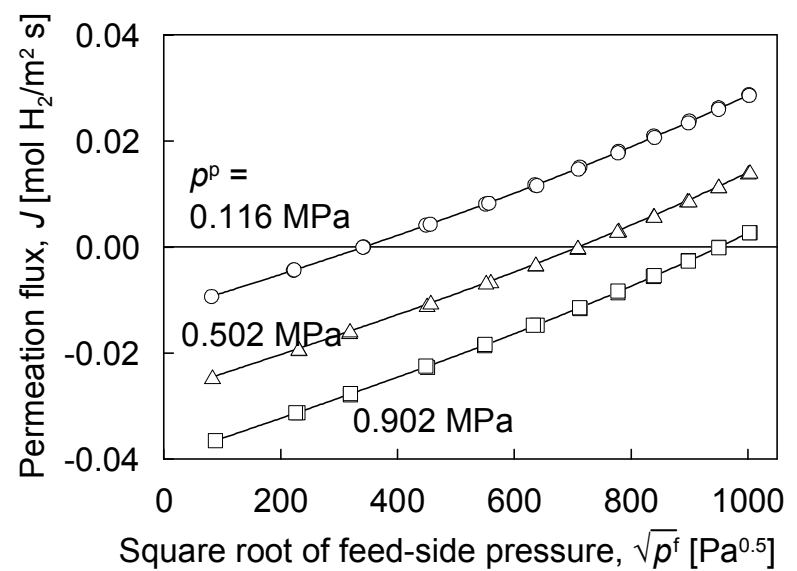

Fig. 3 Hydrogen permeation flux at $573 \mathrm{~K}$ vs. the square root of feed-side pressure for three constant permeation-side pressures, 0.116, 0.502 and $0.902 \mathrm{MPa}$.

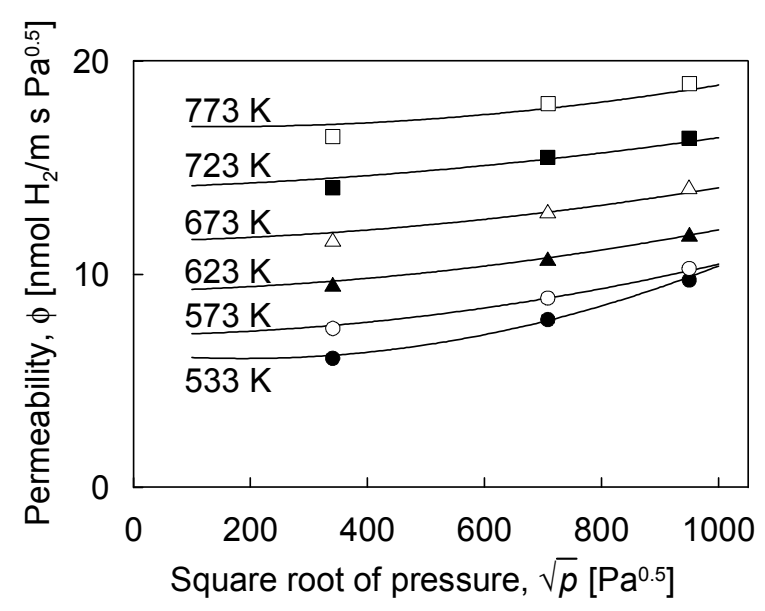

Fig. 4 Hydrogen permeability as a function of the square-root of pressure evaluated using Eqs. 2 and 3.

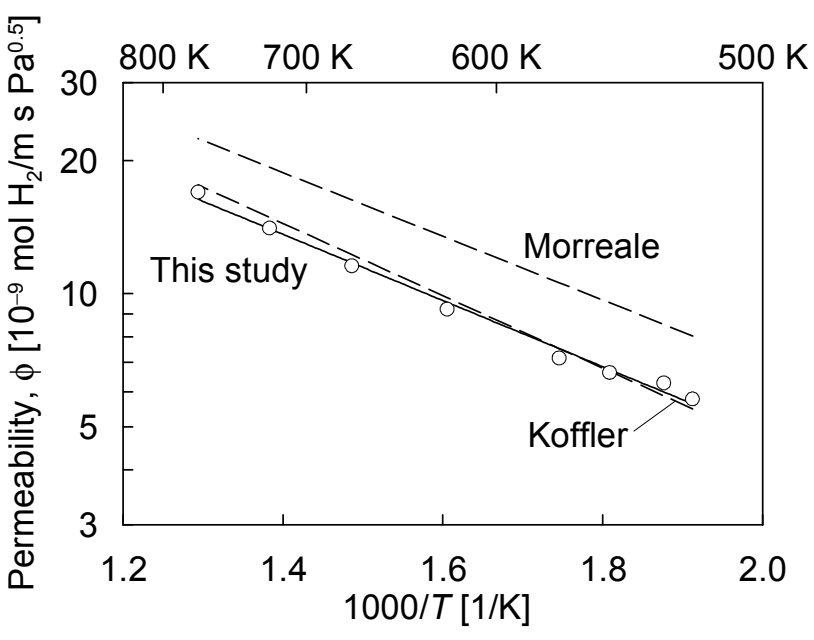

Fig. 5 Arrhenius plot of intrinsic hydrogen permeability compared with diffusion coefficient in the literature[4,8] (broken lines). 
factor and activation energy for intrinsic permeability as $1.52 \times 10^{-7} \mathrm{~mol} \mathrm{H}_{2} / \mathrm{m} \mathrm{s} \mathrm{Pa}^{0.5}$ and $14.3 \mathrm{~kJ} / \mathrm{mol}$, respectively. The figure compares this study with the data reported by the literature. Morreale et al.[4] reports higher permeability but it is probably due to high experimental pressures. Fundamentally, this study is consistent with literature.

As described above, the validity of the extended permeability has been confirmed under wider conditions, i.e., at low pressures and for a thick membrane.

Finally, the resultant parameters are summarized in Table 3. The $c$ represents the intrinsic permeability. The $a$ and $b$ indicate the degree of deviation from the square-root law. The $d$ does not affect permeability but only flux as seen in Eq. 6 . The value is determinable from $a, b, c$, and permeation-side pressure so that the flux is zero when the feed-side pressure is equivalent to the permeation-side pressure. Therefore, $d$ is not shown in the table.

Table 3 Parameters to describe pressure-dependent permeability

\begin{tabular}{|c|c|c|c|}
\hline $\begin{array}{c}\text { Temperature } \\
{[\mathrm{K}]}\end{array}$ & $\begin{array}{l}a \quad\left[10^{-15} \mathrm{~mol}\right. \\
\left.\mathrm{H}_{2} / \mathrm{m} \mathrm{s} \mathrm{Pa}^{1.5}\right]\end{array}$ & $\begin{array}{lcc}b \quad\left[10^{-12}\right. & \mathrm{mol} \\
\left.\mathrm{H}_{2} / \mathrm{m} \mathrm{s} \mathrm{Pa}\right] & \\
\end{array}$ & $\begin{array}{l}c \quad\left[10^{-9} \mathrm{~mol}\right. \\
\left.\mathrm{H}_{2} / \mathrm{m} \mathrm{S} \mathrm{Pa}^{0.5}\right]\end{array}$ \\
\hline 523 & 6.70 & -2.10 & 5.77 \\
\hline 533 & 6.58 & -2.47 & 6.28 \\
\hline 553 & 4.09 & -0.57 & 6.63 \\
\hline 573 & 3.02 & 0.31 & 7.15 \\
\hline 623 & 2.30 & 0.57 & 9.22 \\
\hline 673 & 2.00 & 0.51 & 11.6 \\
\hline 723 & 1.54 & 0.81 & 14.1 \\
\hline 773 & 2.59 & -0.68 & 17.0 \\
\hline
\end{tabular}

\section{Summary}

Hydrogen permeation flux through a palladium membrane $200 \mu \mathrm{m}$ thick did not obey the square-root law completely. From the permeation behavior, pressure-dependent permeability was evaluated. The resultant permeability decreased with decreasing pressure. The pressure dependency was the smaller at the lower pressures and the higher temperatures. Permeability became constant at low pressures. These results confirmed the validity of this extended permeability under wider conditions, i.e., at lower pressures and for a thicker membrane.

\section{References}

[1] A. Winkelmann: Ann. physik. Vol. 6, (1901), p. 104

[2] R .C. Hurlbert and J. O. Konecny: J. Chem. Phys. Vol. 34 (1961), p. 655

[3] N. Itoh, W.-C. Xu, S. Hara, H.-M. Kimura and T. Masumoto: J. Membr. Sci. Vol. 139 (1998), p. 29

[4] B. D. Morreale, M. V. Ciocco, R. M. Enick, B. I. Morsi, B. H. Howard, A. V. Cugini and K. S. Rothenberger: J. Membr. Sci. Vol. 212 (2003), p. 87

[5] T. Nambu, N. Shimizu, H. Ezaki, H. Yukawa and M. Morinaga: J. Jpn. Inst. Metals. Vol. 69 (2005), p. 841

[6] S. Hara, M. Ishitsuka, H. Suda, M. Mukaida and K. Haraya: J. Phys. Chem. B Vol. 113 (2009), p. 9795

[7] R. Dittmeyer, V. Höllein and K. Daub: J. Molec. Catal. A: Chem. Vol. 173 (2001), p. 135

[8] S. A. Koffler, J. B. Hudson and G. S. Ansell: Trans. Metall. Soc. AIME Vol. 245 (1969), p. 1735 